\title{
A DistribUIÇÃO NORMAL E O QUINCUNX ${ }^{+*}$
}

\author{
Ricardo Roberto Plaza Teixeira \\ Centro Federal de Educação Tecnológica de São Paulo \\ Pontifícia Universidade Católica de São Paulo \\ Riama Gouveia Pereira \\ Margareth Yuri Takeuchi \\ Centro Federal de Educação Tecnológica de São Paulo \\ São Paulo - SP
}

\section{Resumo}

Neste trabalho são apresentados os resultados obtidos em sala de aula, em diferentes níveis de ensino, a partir da utilização do "quincunx", dispositivo criado para estudos sobre hereditariedade, numa atividade experimental envolvendo dados quantitativos e qualitativos da Distribuição de Probabilidades Binomial e Normal. A análise parte de uma comparação entre a História da Estatística e a Psicogênese, mostrando as relações que se pode estabelecer entre as etapas de desenvolvimento histórico e cognitivo.

Palavras-chave: Educação; Estatística; Psicogênese; História da Ciência.

\begin{abstract}
This paper presents the results obtained inside the classroom, in different learning levels, with the use of "quincunx", a device created for the study of heredity in an experimental activity involving quantitative and qualitative data of Binomial and Normal Probabilities Distribution. The analysis stems from a comparison between the History of Statistics and Psychogenesis,
\end{abstract}

\footnotetext{
${ }^{+}$The normal distribution and the Quincunx

* Recebido: julho de 2007. Aceito: março de 2008.
} 
showing the relations that can be established between historical and cognitive development phases.

Keywords: Education; Statistics; Psychogenesis; History of Science.

\section{Introdução}

Quanto maior a multidão e maior a anarquia aparente, mais perfeita é sua variação. É a lei suprema da desrazão. Em qualquer lugar onde uma grande amostra de elementos caóticos seja colhida e escalonada segundo a sua magnitude, uma forma de regularidade insuspeitada e das mais belas prova ter estado latente todo o tempo. Os pontos mais altos da fileira escalonada formam uma curva harmoniosa de proporções invariáveis; e cada elemento, ao ser posicionado, encontra como que um nicho predeterminado, cuidadosamente adaptado para contê-lo.

\section{Francis Galton}

Não é de hoje a preocupação com a inserção de atividades experimentais no ensino de ciências. Nos Estados Unidos, as iniciativas governamentais que ocorreram, sobretudo durante a década de 1960, foram um marco importante nesse sentido. Um dos projetos mais famosos desenvolvidos nesse período foi o PSSC (Physical Science Study Committee), composto por livros que tratavam das recentes descobertas da física moderna e por produções audiovisuais, com diversas experiências e demonstrações de muitas teorias.

O PSSC (1960) também chegou ao Brasil, onde foi traduzido e aplicado em escolas de segundo grau, sem, no entanto, alcançar grande sucesso. Seus maiores problemas estavam relacionados à linguagem utilizada nos textos e à falta de adequação à realidade educacional do país.

Mesmo assim, muitos dos experimentos apresentados pelo PSSC - submetidos às modificações necessárias - podem ser de grande utilidade no ensino das ciências da natureza, principalmente quando a simples explicação teórica não permite plena compreensão dos conceitos abordados. Um exemplo de um assunto como esse trabalhado com intensidade pelo PSSC é a Distribuição Normal: dizer que muitos dos fenômenos naturais e sociais seguem um mesmo padrão de probabilidades e que diversos eventos aleatórios se distribuem de acordo com uma função pré-definida não é suficiente para explicar as idéias subjacentes e convencer 
um aluno. Contudo, a compreensão desse comportamento é imprescindível para estudos futuros em muitas áreas de conhecimento como Física, Biologia e Matemática.

Com esse problema em mente, selecionamos a partir de um dos episódios da série produzida pelo PSSC, uma atividade experimental baseada numa mesa com diversas fileiras de pregos e canaletas ao final, cujo nome técnico é "quincunx".

A idéia inicial era a aplicação do equipamento para o estudo da Distribuição Normal com alunos do terceiro semestre do curso de Licenciatura em Física do Centro Federal de Educação Tecnológica de São Paulo (CEFET-SP) de forma a analisar os benefícios, em termos de aprendizado, do uso de atividades práticas com o quincunx em aulas de Estatística. No decorrer do trabalho, o projeto foi ampliado e a atividade estendeu-se ao Ensino Médio e ao Ensino Fundamental. Muito além das expectativas, o experimento trouxe à tona relações entre o desenvolvimento cognitivo e o desenvolvimento histórico de conceitos como aleatoriedade, probabilidade e distribuição normal.

\section{O quincunx}

Francis Galton (1822-1911) (KEYNES, 1993; GILLHAM, 2001) nasceu em Sparbrook, na Inglaterra e foi primo de Charles Darwin. O avô de ambos, Erasmus Darwin, influenciou decisivamente na sua formação, sobretudo pela sua participação na Sociedade Lunar de Birmingham que congregava muitos cientistas, inventores, pesquisadores e livres-pensadores ingleses importantes da época como James Watt - responsável pelo desenvolvimento da máquina a vapor - e Joseph Priestley - responsável pela descoberta do oxigênio conjuntamente com o francês Antoine Lavoisier e o sueco Carl Scheele. Influenciado pelos pais, estudou medicina, mas veio a abandoná-la após terminar o curso. Assim como seu primo, era um viajante entusiasta, tendo sido inclusive premiado pela Sociedade Geográfica Real e pela Sociedade Geográfica Francesa devido aos seus estudos cartográficos na região da atual Namíbia. Realizou estudos nas áreas de geografia, estatística, meteorologia, psicologia, educação, sociologia e antropologia, mas em 1865 seu principal interesse se definiu: a hereditariedade.

A publicação do livro "A origem das espécies" por Darwin, em 1859, influenciou marcadamente as idéias de Galton, que procurou, ao longo do resto de sua vida, estudar as conseqüências da evolução na espécie humana, linha de pensamento com a qual o próprio Darwin era bastante cuidadoso. Seu foco passou a 
ser a forma como habilidades humanas - inclusive as intelectuais - são ou não são herdadas; para isto, ele realizou estudos e levantou dados biométricos com o objetivo de separar as influências ambientais das características hereditárias, iniciando o debate "nature versus nurture" que se estende até hoje e que, para muito além da sua característica científica, tem conseqüências políticas óbvias. Os seus estudos, conjuntamente com os realizados por Pearson e Fisher, estabeleceram o campo de conhecimento da bioestatística. Particularmente, foi Galton quem propôs originalmente a idéia de regressão e correlação entre diferentes tipos de dados.

Em 1863, após conhecer os escritos de Quetelet, imbuiu-se instantaneamente da universalidade da distribuição normal. O modo como a utilizou, contudo, foi muito diferente do que Quetelet defendia. Galton viu a distribuição normal como um método de classificar dados em grupos de diferentes origens. Dalton criou a polêmica idéia de "eugenia" - o melhoramento racial das gerações seguintes - que teve ao longo do século XX uma triste história, já que foi apropriada por ideólogos nazistas e racistas.

Em 1873-1874, Galton projetou um curioso aparelho experimental conhecido como "quincunx" (Figura 1) ou também "Galton's board" (placa ou jogo de Galton). Essa máquina era um engenhoso modelo físico da teoria dos erros, a qual ele acreditava ser aplicável a muitos fenômenos no campo da Biologia e da Física. Encerrada atrás de um vidro, havia uma seção transversal de um funil que se abria para um arranjo de pinos de metal dispostos a intervalos iguais, com compartimentos verticais abaixo dos pinos. Ao cair pelo funil, os chumbinhos de espingarda (ou bolinhas) se distribuiriam, aleatoriamente, para a direita ou para a esquerda pelos espaços entre os pinos que representavam, na teoria de Galton, as perturbações aleatórias independentes da natureza. No final do processo, eles se acumulavam nos compartimentos inferiores em pilhas que lembram uma curva normal. Galton chamou esse fenômeno de lei do desvio. Ele acreditava que as causas que atuavam sobre uma característica herdada, tais como a altura, eram um "exército de influências perturbadoras insignificantes" (representadas pelos pinos) e que a lei do desvio genético era puramente numérica e seguia universalmente a lei genérica da distribuição normal. Dispositivos semelhantes ao quincunx podem ser vistos em museus científicos. Em alguns lugares eles são enormes, com bolas de tênis no lugar das bolinhas. Há alguns sítios na internet com simuladores de um quincunx de forma a visualizar a distribuição que se obtém com este aparelho; um destes sítios, que pode ser usado em simulações durante aulas de estatística, é:

$<$ http://www.mathsisfun.com/probability/quincunx.html $>$. 


\section{Metodologia e resultados}

O quincunx utilizado no estudo experimental envolvendo dados quantitativos e qualitativos da Distribuição Binomial e da distribuição Gaussiana consiste num conjunto de bolinhas que descem por uma rampa com uma certa inclinação; durante o seu percurso estas bolinhas colidem com os pregos colocados ao longo da rampa. Não é difícil imaginar condições nas quais as bolinhas têm igual probabilidade de desviar para a esquerda ou para a direita de um prego. Se por baixo de cada prego estão colocados dois pregos numa linha horizontal e o declive da rampa estiver corretamente ajustado, a bolinha baterá num ou noutro depois de desviar do primeiro prego. Novamente, a bolinha deve ter igual probabilidade de queda à esquerda ou à direita desses pregos. Após o segundo choque, as probabilidades de queda à esquerda de ambos os pregos ou entre eles ou à direita de ambos, deveria estar na proporção 1:2:1. O processo pode ser continuado e está claro que as probabilidades de uma bolinha passar entre os diferentes pregos de um fila são proporcionais aos números do Triângulo de Pascal (Fig. 1).
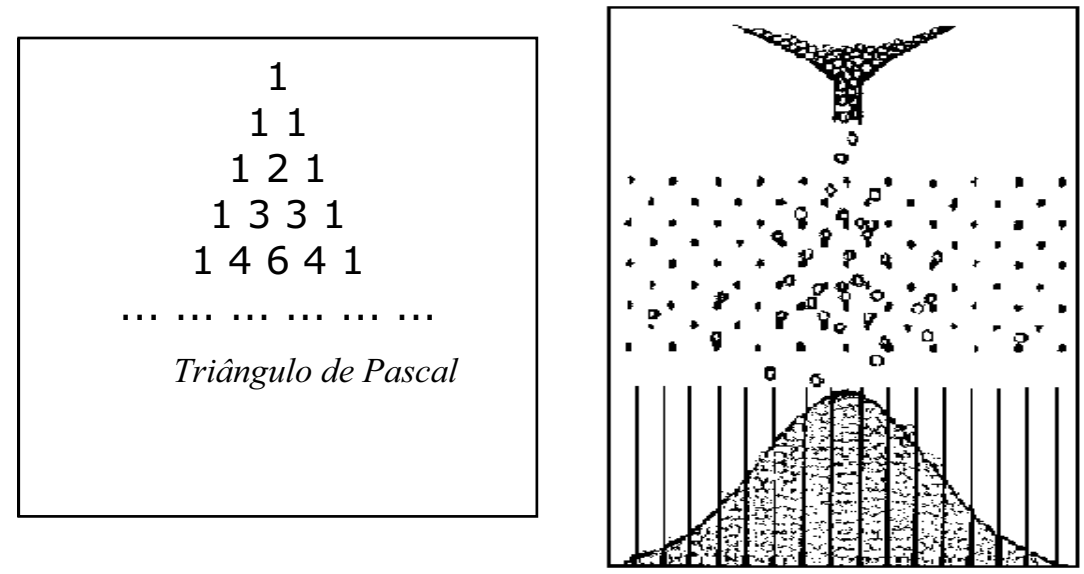

Fig. 1 - O triângulo de Pascal (à esquerda) é uma distribuição estatística obtida a partir de um "aparelho" como o quincunx (à direita) proposto por Francis Galton (1889).

Para estudarmos o comportamento estatístico, construímos efetivamente um quincunx; na base da rampa foram feitas divisões para as bolinhas e colocado um pedaço de acrílico para que as mesmas não passem de uma divisão para outra. $\mathrm{Na}$ parte superior da rampa foi construído um reservatório para colocar as boli- 
nhas. Quando as bolinhas descem pela rampa abaixo, são desviadas pelos pregos, que se encontram distribuídos de forma conveniente. Se o ângulo for ajustado adequadamente, o número de bolinhas nos compartimentos pode aproximar-se muito da distribuição binomial. A curva formada pelas colunas de bolinhas nos compartimentos dá uma idéia da forma da distribuição normal.

No caso do quincunx, a variável que está em jogo é do tipo discreta, mais especificamente a canaleta na qual a bolinha irá cair. Nesse caso, emprega-se a distribuição binomial para descrever o problema. A binomial é usada quando se deseja saber a probabilidade de obter-se $\mathrm{x}$ sucessos em $\mathrm{n}$ tentativas se a probabilidade de obter-se sucesso em uma tentativa é p e conseqüentemente a probabilidade de obter-se fracasso em uma tentativa é q=1-p. Portanto, em cada tentativa ou experimento individual só dois resultados são possíveis, que são denominados de sucesso ou fracasso: o raciocínio subentendido é binário. A distribuição de probabilidade binomial $\mathrm{P}(\mathrm{x})$ é então dada por:

$$
P(x)=\frac{n !}{x !(n-x) !} p^{x} q^{n-x}=\left(\begin{array}{l}
n \\
x
\end{array}\right) p^{x} q^{n-x}
$$

Prova-se que a distribuição binomial $\mathrm{P}(\mathrm{x})$ pode ser aproximada por uma curva normal com média $\mu=$ np e variância $\sigma^{2}=$ npq.

Sendo $\mathrm{x}$ uma variável contínua, o que é relevante é a integral desta função entre quaisquer dois valores $\mathrm{x}_{1}$ e $\mathrm{x}_{2}$ que fornece a probabilidade de, aleatoriamente, um valor qualquer de $\mathrm{x}$ estar dentro deste intervalo. Muitos livros de estatística apresentam em seus apêndices tabelas com os valores padronizados de muitas destas integrais. É importante destacar, finalmente, que a integral desta função entre $-\infty$ e $+\infty$ será necessariamente igual a 1 (ou 100\%) que é a probabilidade de que $\mathrm{x}$ tenha qualquer valor real possível. Nesse caso, a função $\mathrm{f}(\mathrm{x})$ é uma distribuição contínua de probabilidades dada pela função de densidade de probabilidade normal ou gaussiana:

$$
f(x)=\frac{1}{\sigma \sqrt{2 \pi}} e^{-(x-\mu)^{2} / 2 \sigma^{2}}, \text { onde }-\infty<\mu<+\infty \text { e } \sigma>0 .
$$

Nesse caso, $\mu$ é a média e $\sigma$ é o desvio padrão. A variável aleatória contínua $\mathrm{x}$ pode variar de $-\infty$ até $+\infty$.

Uma situação especial e didática é o caso da distribuição normal reduzida para a qual a média $\mu=0$ e o desvio padrão $\sigma=1$. De modo genérico, usando a variável reduzida $\mathrm{z}=(\mathrm{x}-\mu) / \sigma$, temos:

$$
f(z)=\frac{1}{\sqrt{2 \pi}} e^{-z^{2} / 2}
$$


É possível facilmente perceber o caráter assintótico de f(z) que tende a zero quando $\mathrm{z}$ tende $\mathrm{a}-\infty$ ou a $+\infty$; é fácil notar também que esta função será simétrica em relação a $\mathrm{z}=0$.

Uma atividade com o quincunx construído pela equipe foi aplicada a diferentes níveis de ensino, apresentando resultados bastante significativos. O primeiro estudo foi realizado com uma turma do Terceiro Semestre do Curso de Licenciatura em Física do Centro Federal de Educação Tecnológica de São Paulo (CEFETSP), no Componente Curricular "Estatística Aplicada à Ciência e à Educação". Os alunos já haviam recebido conhecimento prévio sobre probabilidade, principalmente no que se refere à distribuição normal. Em um segundo momento, a atividade foi realizada com alunos da $5^{\text {a }}$ e $6^{\text {a }}$ séries do Ensino Fundamental, em uma escola da rede pública estadual na cidade de São Paulo. Sem conhecimento acadêmico sobre o assunto, os dados obtidos permitem uma noção acerca das concepções e conhecimentos prévios dos estudantes. A terceira etapa foi realizada com alunos do Ensino Médio, em turmas de primeiro e terceiro ano, na mesma escola estadual citada anteriormente. Apesar de se encontrarem num estágio escolar mais avançado, os alunos não haviam recebido nenhuma instrução teórica sobre distribuição de probabilidades.

Para cada grupo de estudantes foi desenvolvido um trabalho específico. Os alunos da Licenciatura em Física receberam uma apostila, com procedimento experimental, questões e análise de dados. No Ensino Fundamental e Médio foi feita uma demonstração prática do equipamento e fornecida uma breve explicação sobre o fenômeno. Nos três casos, no entanto, foi solicitado aos alunos que respondessem a um questionário antes da atividade, com perguntas sobre as estimativas deles sobre o que aconteceria nos experimentos; após os experimentos, os alunos foram estimulados a comparar as suas estimativas com os dados realmente encontrados. Esse procedimento mostrou-se pedagogicamente interessante, já que permitiu que os estudantes formulassem modelos explicativos e testassem imediatamente estes modelos confrontando suas concepções espontâneas com a realidade.

Os alunos do curso superior, já com um instrumental teórico maior, responderam às questões mais precisamente, e com melhores argumentos, sendo capazes de emitir opiniões mais amparadas em argumentos lógicos. Todos os alunos desta etapa previram uma distribuição de probabilidades simétrica, na forma da curva do sino (figura 2a) da distribuição normal.

No Ensino Médio, a percepção da aleatoriedade do lançamento de uma única bolinha foi percebida, mas a formação de um padrão de distribuição regular no conjunto de lançamentos não foi prevista por aproximadamente metade dos 
grupos. Nos grupos em que uma regularidade foi imaginada, as formas sugeridas foram principalmente o triângulo (Fig. 2b) e o semicírculo (Fig. 2c), existindo apenas um desenho no formato retangular (Fig. 2d).

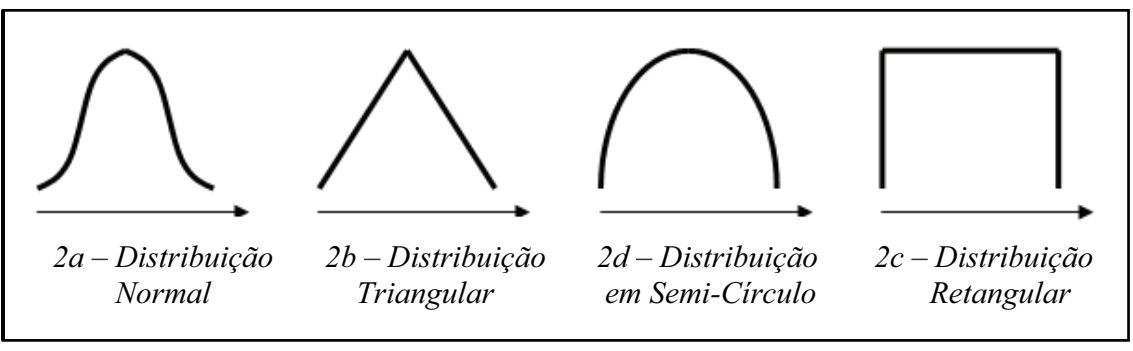

Fig. 2 - Formas esquemáticas possíveis para a distribuição das bolinhas sugeridas pelos alunos antes da realização do experimento.

Para os estudantes do Ensino Fundamental, as respostas partiam de tentativas concretas de encontrar um "caminho" preferencial que seria seguido pelas bolinhas, não sendo cogitada a idéia do evento aleatório. Muitos foram os grupos que previram um mesmo número de bolinhas em cada canaleta, construindo um retângulo (Fig. 2d), mas existiram também sugestões de distribuições triangulares (Fig. 2b) e em semicírculo (Fig. 2c). Algumas poucas distribuições não eram regulares, e foram então denominadas pelos alunos de "tudo diferente".

Apesar dessas diferenças, foi possível notar um fato bastante interessante: nos três casos - na verdade, com uma só exceção - as estimativas para a porcentagem de bolinhas que cairiam tanto na canaleta central quanto nas canaletas da extremidade esquerda foram, em geral, superiores aos valores efetivamente encontrados. Na canaleta da extremidade, os alunos do ensino superior foram aqueles que fizeram as estimativas mais próximas dos resultados obtidos, o que era esperado devido aos conhecimentos prévios desses estudantes.

Os valores superestimados, tanto para a canaleta central quanto para as canaletas laterais, contrariam a lógica e a "conservação de bolinhas", que exigiriam a compensação de um excesso no centro com a redução dos valores na lateral ou a diminuição dos valores centrais para compensação de excedentes na lateral. Portanto, ou se está superestimando o número de bolinhas ou se está subestimando o número de canaletas.

Um fato interessante foi a existência de vários alunos que insistiram em uma distribuição em forma de U (ou seja, uma curva do sino invertida) com mais bolinhas caindo nas extremidades do que no centro, usando o argumento de que, quando a bolinha em um prego desvia para um lado, ela desviará sistematicamente 
para o mesmo lado nas próximas colisões com as séries seguintes de pregos. Conforme observamos, parece existir um tipo de raciocínio de que há um "caminho diagonal" privilegiado, pois nas diagonais que formam $45^{\circ} \mathrm{com}$ as canaletas não haveria pregos para impedir o movimento e as bolinhas acabariam se conduzindo naturalmente para as canaletas mais externas. Esse raciocínio, em certo sentido determinista, revela tanto uma dificuldade na elaboração de raciocínios probabilísticos, nos quais o acaso desempenha um papel fundamental - já que o resultado final deve-se à combinação de sucessivas mudanças aleatórias de trajetória - quanto um desconhecimento da maneira como a componente da força da gravidade paralela ao plano do quincunx atua sobre as bolinhas.

Um paralelo interessante aqui pode ser traçado com uma dificuldade existente no ensino da evolução das espécies: os processos durante a evolução também são submetidos às leis do acaso - sobretudo no que diz respeito às mutações, que são essenciais - mas, ao contrário do quincunx, são conduzidos também por um fator externo que é a seleção natural. Por exemplo, há um argumento muito disseminado de oposição à teoria da evolução que diz respeito, por exemplo, à probabilidade diminuta de que por acaso todos os componentes de um olho aparecessem em uma espécie de um ser vivo (DAWKINS, 2001) - seria aqui como se todas as bolinhas fossem cair em uma canaleta lateral, por exemplo. É claro que, se as bolinhas só estão submetidas ao acaso, isto é extremamente improvável, mas no caso dos seres vivos há um agente externo que é a seleção natural: nos predadores foram desenvolvidos olhos cada vez melhores de modo que eles conseguissem caçar melhor, se alimentar melhor e ter mais descendentes; nas presas, olhos melhores foram desenvolvidos, de modo que elas fossem menos caçadas, sobrevivessem mais e tivessem mais descendentes. Em ambos os casos, há uma pressão seletiva por olhos melhores: esta pressão atua ao longo de muitas - milhares de - gerações, fazendo com que aquilo que, de acordo com o acaso, fosse muito improvável, se materializasse no olho de uma águia, por exemplo. No caso do quincunx, essa pressão externa pode ser representada quando viramos o quincunx, de modo que a força da gravidade comece a atuar de modo assimétrico, fazendo com que um número maior de bolinhas efetivamente caiam mais nas canaletas próximas de um lado do que nas canaletas centrais.

Os experimentos realizados permitiram concluir que a forma da distribuição normal não é intuitiva ou espontânea para os alunos e que é importante trabalhar seus conceitos prévios se o objetivo for a construção de uma aprendizagem efetiva dos conceitos envolvidos. Para isso, a História da Ciência parece fornecer importantes analogias. 


\section{Analogias entre história e psicogênese}

Um olhar mais atento para os resultados obtidos permitiu-nos notar que há uma intensa relação entre a história da estatística e o desenvolvimento cognitivo dos adolescentes e jovens pesquisados sobre esse tema.

Desde a Grécia até a Idade Média, foi dominante a idéia de que todos os fenômenos possuíam uma causa determinada. Partindo de Leucipo e Demócrito, por volta do ano 450 a.C., com a primeira teoria do átomo, passando por Crísipo, um estóico que viveu do ano 280 até o ano 207 a.C., e chegando à visão determinista defendida por Thomas Hobbes (1588-1679) de que todos os eventos eram predeterminados por Deus, durante a Idade Média, o determinismo não deixou muito espaço para a aleatoriedade.

A partir de um debate com Hobbes em 1646, o bispo de Derry, John Bramhall, apresentou a possibilidade da indeterminação dos eventos ser causada por contingências. Oito anos após, em 1654, a correspondência trocada por Pascal e Fermat iniciou os primeiros estudos sobre probabilidade. Era trazida à tona a incerteza, que logo seria abafada por uma grande descoberta: a física newtoniana. A capacidade de previsão da mecânica clássica reforçou o ponto de vista determinista, fazendo-o permanecer em destaque até o século XX, quando a revolução científica proporcionada pela Mecânica Quântica levou à construção de um novo paradigma não-determinístico na Física.

Entretanto, ironicamente, foi a própria teoria de Newton que, posteriormente, deu impulso à descoberta das distribuições de probabilidade. Para comprovar a teoria era necessário aplicá-la a situações práticas, realizando medidas e comparando-as com as previsões. Efetuando as medições, os cientistas - sobretudo os astrônomos - verificaram que, por mais precisos que fossem os instrumentos e por mais cuidadosas que fossem as observações, os valores medidos eram sempre ligeiramente diferentes. Nasceu então a Teoria dos Erros.

Inicialmente, acreditava-se que os erros de uma medida seguiam o mesmo padrão que as probabilidades de resultados no lançamento de um dado, e que estas possibilidades estariam uniformemente distribuídas, numa forma que poderíamos chamar de retangular (DENNETT, 2003). Na prática, porém, não é isso que acontece, e os erros concentram-se no meio da distribuição, decaindo em direção às laterais. Num segundo momento, Thomas Simpson, em um estudo datado de 1756, atribuiu à distribuição de probabilidades nos erros de medida a forma triangular. Chegou-se a esta conclusão através da comparação dos erros de observações astronômicas com os resultados obtidos da soma das faces de dois dados lançados; neste caso, os diferentes valores para a soma (os números naturais entre 2 e 12) 
não são eqüiprováveis: o valor mais provável para a soma é 7 (que está no centro como no caso do quincunx) e os valores menos prováveis para a soma são 2 e 12 (que estão nas laterais, também como no quincunx). Quase ao mesmo tempo, Daniel Bernoulli em 1777 propôs a idéia de que a distribuição de erros fosse análoga aos desvios das flechadas de um arqueiro. A partir desse raciocínio, chegou-se a uma curva de probabilidade com formato semicircular, com o raio estando relacionado ao maior erro cometido; a propósito, ainda hoje, é comum os alunos associarem equivocadamente a idéia de desvio padrão a este "maior erro cometido".

Poucos anos depois, foram aplicadas bases científicas a essas teorias e foi então desenvolvida a curva normal de probabilidades, em paralelo, por dois grandes matemáticos da época: o francês Pierre Simon - o marquês de Laplace - e o alemão Carl Friedrich Gauss. Devido aos trabalhos de Gauss, a distribuição normal também é conhecida como distribuição gaussiana (MILONE, 2004).

Nas respostas dos estudantes em nossa pesquisa, muitos paralelos foram percebidos entre o amadurecimento conceitual individual dos alunos sobre a distribuição obtida no quincunx e a evolução ocorrida no desenvolvimento histórico da estatística. Muitos dos alunos mais jovens atribuíam ao fenômeno uma causalidade, tentando prever o "caminho" de cada bolinha e acreditando que uma reprodução do experimento apresentaria resultado idêntico. Outros, na mesma faixa etária, pensavam numa distribuição eqüiprovável, a que davam o nome de retangular. A maioria dos estudantes que pertenciam ao Ensino Médio reconheceram a aleatoriedade, e sugeriram as formas triangulares e o semicírculo para a distribuição de probabilidades. No Ensino Superior, a Matemática foi usada como guia e as previsões buscaram apoio na teoria científica e nos conceitos que já tinham sido trabalhados em aula. Na mesma linha de argumentação proposta por Jean Piaget e Rolando Garcia (1983), encontraram-se muitas similaridades de mecanismos entre os desenvolvimentos psicogenéticos - referentes à aprendizagem e à construção do conhecimento pelas crianças e pelos jovens - e os desenvolvimentos sociogenéticos - referentes à evolução das idéias científicas ao longo da história. Assim, a evolução do conhecimento, tanto individual quanto socialmente, apresenta grandes e, de certa maneira, pedagógicos paralelos.

Outra analogia pode ainda ser traçada entre a história da Estatística e a psicogênese. Com base nos estudos de Piaget e Garcia, pode-se acrescentar uma relação que não se refere aos fatos propriamente ditos, mas a etapas de desenvolvimento que se apresentam em ambos os casos. Tanto na construção dos conceitos de Estatística ao longo da história quanto nas etapas de desenvolvimento cognitivo dos estudantes, percebe-se a passagem de uma fase mais concreta, que apresenta 
apenas abstrações empíricas, para uma fase mais elaborada, que oferece também abstrações reflexivas.

Na história, as primeiras teorias surgem de analogias com fatos concretos, como a jogada de dados ou as flechas de um arqueiro. Os estudantes mais jovens também necessitam da experiência concreta e elaboram maneiras de testar o equipamento para verificar o destino das bolinhas.

Em ambos os casos - aprendizagem individual e história social - em um segundo momento, as teorias são formuladas com base em conceitos prévios, com uma ligação menos intensa com o que pode ser diretamente observado: no desenvolvimento dos jovens apareceu primeiro a idéia do evento aleatório e posteriormente a compreensão do modelo matemático que o explicava - a distribuição em forma de sino.

Finalmente, é interessante lembrar que esse debate entre determinismo e acaso atravessou o século XX mesmo na Física Moderna, contrapondo dois grandes cientistas, Albert Einstein e Niels Bohr, no que diz respeito às suas interpretações sobre a Física Quântica (STEWART, 1991).

\section{Conclusões}

A estatística é a tentativa de matematização da aleatoriedade, de forma a nos aproximar do objeto do conhecimento que, neste caso, é constituído por todos os fenômenos - como os observados no quincunx - que se devem ao acaso e apresentam características randômicas. Essa matematização evoluiu ao logo da história, passando por várias etapas diferentes de sistematização.

Charles Darwin (2000), no capítulo com as conclusões de seu livro "A origem das espécies" já alertava no século XIX: "a analogia pode ser um guia enganador". Portanto, é preciso sempre ter em mente os limites das analogias verificadas. Mas o próprio Darwin utilizou-se muito de analogias em seus argumentos. No campo da própria Biologia, Ernst Heinrich Haeckel (1992), influenciado pelas idéias darwinistas, defendeu, ainda no século XIX, que a ontogênese - o desenvolvimento do indivíduo orgânico - seria a recapitulação da filogênese - o desenvolvimento da linhagem ou do filo ao qual ele pertence.

Um raciocínio semelhante ocorre quando traçamos paralelos entre a História da Ciência e a Psicogênese do Conhecimento e constatamos a existência de grandes correspondências entre a produção histórica coletiva do conhecimento (sociogênese) e a produção psicogenética individual do conhecimento. A história de uma idéia ou conceito em geral fornece indícios sobre o real significado desta 
idéia ou conceito no contexto em que surgiu e isto tem importantes conseqüências pedagógicas. Alguns trabalhos recentes (CARVALHO, 1993; FERNANDES, 1998; FRANCO, 1995) discutem a necessidade de reconhecer o valor da história para a compreensão de como as crianças e os jovens constroem o conhecimento. Mas as etapas do saber, ao contrário do senso comum, muitas vezes não se sucedem de forma linear, tanto na história da ciência quanto no desenvolvimento individual dos estudantes. Além disso, em ambos os casos, cada período inicia-se pela reorganização das idéias que herdou do período anterior: "O parentesco entre a epistemologia histórico-crítica e a epistemologia genética está não apenas nas interações elementares entre sujeitos (indivíduos ou sociedades) e objetos, mas sobretudo no modo como um nível anterior condiciona a formação do seguinte" (PIAGET, 1983).

Todo conhecimento, por mais novo que seja, não é um primeiro fato totalmente independente daqueles que o precederam: somente está completa a análise da evolução de um conceito se levarmos em conta o papel dos seus precursores. Dessa forma, torna-se essencial a caracterização dos grandes períodos sucessivos do desenvolvimento de um conceito. As normas científicas situam-se no prolongamento de práticas anteriores de pensamento às quais são incorporadas duas novas exigências: coerência lógica interna e verificação experimental. O desenvolvimento cognitivo, tanto na história da ciência quanto na história do indivíduo, caracteriza-se pela assimilação e acomodação das novidades às estruturas precedentes.

A importância da utilização de um equipamento experimental como o quincunx em aulas de estatística para o estudo de distribuições de probabilidades, permite também estabelecer analogias entre o processo histórico de desenvolvimento do campo da estatística e a construção desses conceitos pelos estudantes do Ensino Fundamental ao Superior. A simplicidade da parte experimental do quincunx, que não demanda a utilização de laboratórios bem equipados, torna esta proposta viável para escolas sem infra-estrutura laboratorial, pois os experimentos podem ser realizados na própria sala de aula. Além disso, o tempo necessário para preparar a atividade não é longo, o que deve estimular a sua aplicação. A visualização da curva do sino, que é construída em segundos pelas bolinhas que caem nas canaletas a partir da aleatoriedade de suas colisões com os pregos, é fundamental na compreensão pelos estudantes de muitas idéias sobre distribuições de probabilidades. As atividades experimentais, utilizando esse equipamento, podem ser empregadas didaticamente para favorecer a articulação entre teoria e prática e introduzir conceitos estatísticos em todos os níveis de ensino, atendendo às recomenda- 
ções dos Parâmetros Curriculares Nacionais para a Educação Básica. Partindo da análise das relações entre a história e a psicogênese, pode-se também construir um plano de curso que inclua metodologias e problemas adequados a cada fase de desenvolvimento, permitindo uma aprendizagem significativa de conceitos estatísticos e probabilísticos em todas as etapas da educação.

\section{Referências}

CARVALHO, A. M. P. C. et al. A história da ciência, a psicogênese e a resolução de problemas na construção do conhecimento em sala de aula. Revista da Faculdade de Educação, São Paulo, v. 19, n. 2, 1993.

DARWIN, C. A Origem das espécies. São Paulo: Editora Hemus, 2000.

DAWKINS, R. O relojoeiro cego. São Paulo: Companhia das letras, 2001.

DENNETT, D. J. Aleatoriedade. São Paulo: Martins Fontes, 2003.

FERNANDES, J. N. Paralelismo entre história e psicogênese da escrita do ritmo musical. Psicologia, São Paulo, v. 9, n. 2, 1998.

FRANCO, C. O modelo piagetiano para a relação entre psicogênese e história da ciência funciona? In: SIMPÓSIO NACIONAL DE ENSINO DE FÍSICA, XI, Niterói, 1995. Atas...

GALTON, F. Natural Inheritance. London: MacMillan, 1889.

GILlHAM, N. W. A life of Sir Francis Galton. Oxford USA Professional, 2001.

HAECKEL, E. H. The riddle of the universe. Prometheus Books, 1992.

KEYNES, M. Sir Francis Galton. London: MacMillan, 1993.

MILONE, G. Estatística geral e aplicada. São Paulo: Thomson, 2004.

PIAGET, J.; GARCIA, R. Psicogénese e História das Ciências. Lisboa: Dom Quixote, 1983.

PSSC. Física. São Paulo: EDART, 1960.

STEWART, I. Será que Deus joga dados? Rio de Janeiro: Jorge Zahar Editor, 1991. 